\title{
Downregulation of YEATS4 by miR-218 sensitizes colorectal cancer cells to L-OHP-induced cell apoptosis by inhibiting cytoprotective autophagy
}

\author{
QIANG FU $^{1 *}$, JING CHENG $^{3 *}$, JINDAI ZHANG $^{1}$, YONGLEI ZHANG ${ }^{1}$, \\ XIAOBING $\mathrm{CHEN}^{2}$, JIANGUO XIE ${ }^{1}$ and SUXIA LUO ${ }^{2}$

\begin{abstract}
Departments of ${ }^{1}$ Gastrointestinal Surgery, ${ }^{2}$ Digestive Oncology, Tumor Hospital of Henan Province
(The Affiliated Tumor Hospital of Zhengzhou University), Zhengzhou, Henan 450008;

${ }^{3}$ Department of Medical Oncology, Zhengzhou Central Hospital (The Affiliated Central Hospital

of Zhengzhou University), Zhengzhou, Henan 450007, P.R. China
\end{abstract}

Received April 26, 2016; Accepted June 10, 2016

DOI: 10.3892/or.2016.5195

\begin{abstract}
Colorectal cancer (CRC) is a leading cause of cancer-related death worldwide. Deregulation of microRNAs (miRNAs) has been reported to participate in CRC progression. In the present study, we observed downregulation of miR-218 and upregulation of YEATS domain containing 4 (YEATS4) in CRC tissues and in multidrug-resistant HCT-116/L-OHP cells compared with these levels in normal tissues and parental HCT-116 cells, respectively. The results indicated that miR-218 overexpression significantly decreased the $\mathrm{IC}_{50}$ value of oxaliplatin (L-OHP) in the HCT-116/L-OHP cells, and suppression of miR-218 significantly enhanced the $\mathrm{IC}_{50}$ of L-OHP in the HCT-116 cells. Flow cytometric analysis showed that miR-218 overexpression alone promoted cell apoptosis in the HCT-116/ L-OHP cells, which was further enhanced in response to L-OHP, and miR-218 inhibition decreased cell apoptosis in the HCT-116 cells following treatment with L-OHP. Western blot analysis indicated that, compared with the small increase observed in HCT-116 cells, the relative LC3 II level
\end{abstract}

Correspondence to: Dr Jianguo Xie, Department of Gastrointestinal Surgery, Tumor Hospital of Henan Province (The Affiliated Tumor Hospital of Zhengzhou University), 127 Dongming Road, Zhengzhou, Henan 450008, P.R. China

E-mail: jianguo_xie@126.com

${ }^{*}$ Contributed equally

Abbreviations: CRC, colorectal cancer; YEATS4, YEATS domain containing 4; L-OHP, oxaliplatin; CQ, chloroquine; miRNAs, microRNAs; DMEM, Dulbecco's modified Eagle's medium; ATCC, American Type Culture Collection; FBS, fetal bovine serum; qRT-PCR, quantitative real-time PCR; UTR, untranslated region; DMSO, dimethyl sulfoxide; LC3, microtubule-associated protein 1 light chain 3

Key words: miR-218, YEATS4, colorectal cancer, chemosensitivity, autophagy, cell apoptosis in HCT-116/L-OHP cells after lysosome inhibition via chloroquine (CQ) was markedly upregulated following L-OHP treatment, suggesting induction of autophagy. Exposure of HCT-116/L-OHP cells to L-OHP after control mimic transfection increased autophagic flux, as reflected by increased LC3 II levels, while miR-218 overexpression partly reversed L-OHP-mediated LC3 II accumulation. Additionally, both miR-218 overexpression and CQ treatment promoted L-OHPinduced HCT-116/L-OHP cell apoptosis. Molecularly, our results confirmed that miR-218 directly targets the YEATS4 gene and inhibits YEATS4 expression. Furthermore, YEATS4 overexpression without the 3'-untranslated region (3'-UTR) restored miR-218-inhibited YEATS4 and LC3 II expression, and abolished miR-218-stimulated cell viability loss and cell apoptosis increase in response to L-OHP. In conclusion, miR-218 sensitized HCT-116/L-OHP cells to L-OHP-induced cell apoptosis via inhibition of cytoprotective autophagy by targeting YEATS4 expression.

\section{Introduction}

Colorectal cancer (CRC) is one of the most common cancers worldwide and the second leading cause of cancerrelated death, despite improvements in available treatment options $(1,2)$. Chemotherapy is consistently used as a palliative or adjuvant treatment, but nearly all patients ultimately develop drug resistance, which has become one of the major causes of CRC recurrence. Additionally, advanced CRC is often resistant to such chemotherapy, resulting in poor outcomes. Studies have proposed many mechanisms as being responsible for drug resistance, including limitation of drug accumulation within cells by reducing uptake, enhancing efflux or affecting membrane lipids; activation of detoxification; alterations in cell cycle checkpoints; and inhibition of cell apoptosis (3). However, only a few predictive and prognostic biomarkers have been validated clinically despite enormous efforts (3), and the exact mechanisms remain unknown. Therefore, it is essential to better understand the underlying mechanisms of chemoresistance, and to uncover novel methods to improve 
the efficacy of chemotherapeutic treatment by targeting these mechanisms.

More effective chemotherapeutic agents, such as oxaliplatin (L-OHP), 5-fluorouracil, irinotecan and the monoclonal antibodies cetuximab, panitumumab and bevacizumab, have been used in clinical practice during the last two decades, but one of the major issues of CRC treatment is the inherent or acquired resistance to therapeutic approaches. L-OHP is a chemotherapeutic agent that is widely used in CRC treatment; however, L-OHP chemoresistance poses a great challenge when treating chemorefractory patients. The treatment of increasing numbers of metastatic CRC patients to L-OHP, as well as its introduction into the adjuvant therapy of early-stage $\mathrm{CRC}$, has led to efforts to identify and target the mechanisms of chemoresistance to L-OHP. Currently, although the molecular mechanisms of the chemoresistance of CRC are not well known, several findings suggest that targeting autophagy may be considered as a potential therapeutic strategy to address chemoresistance in the treatment of CRC (4-6). In the present study, we investigated the mechanisms involved in cell autophagy underlying L-OHP-induced cell apoptosis and chemoresistance in CRC cells, the knowledge of which may contribute to clinical treatment.

MicroRNAs (miRNAs) are a class of small, endogenous, non-coding, single-stranded RNAs, which have been recognized as key regulators of gene expression at posttranscriptional levels. miRNAs are broadly involved in tumor proliferation, invasion, angiogenesis and drug resistance (7), which may add new dimensions to the diagnosis and treatment of cancer. Several miRNAs are known to be dysregulated in CRCs and have been linked to biological processes involved in tumor progression and response to anticancer therapies $(8,9)$. In particular, decreased miR-218 expression has been observed in CRC tissues, and patients with low miR-218 expression have shorter survival times; furthermore, an increased level of serum miR-218 was noted one month after surgery (10). Thus, we infer that miR-218 may play important roles in the development and progression of CRC, which may serve as a potential diagnostic and prognostic biomarker of CRC.

YEATS domain containing 4 (YEATS4) is a highly conserved nuclear protein that was originally identified in human glioma (11). Research has shown that YEATS4 functions as an oncogene and is amplified in various types of malignancy, including CRC (12). Transcriptional networks isolated from the tissues of drug-resistant patients suggest that YEATS4 could be targeted to ameliorate drug resistance (13). In addition, several studies have shown that therapy-induced autophagy may be a novel mechanism of resistance to anticancer agents $(14,15)$. In the present study, we demonstrated that YEATS4 is a downstream target of miR-218 and that miR-218 sensitizes CRC cells to L-OHP-induced cell apoptosis by inhibiting cytoprotective autophagy.

\section{Materials and methods}

Tissue samples and cell culture. Human CRC and the corresponding non-tumor colon samples were collected at the time of surgical resection at the Tumor Hospital of Henan Province (The Affiliated Tumor Hospital of Zhengzhou University). The present study was conducted following patient informed consent in accordance with the Declaration of Helsinki and was approved by the Ethics Committee of Zhengzhou University. The samples were immediately snap-frozen and stored in liquid nitrogen. Samples used for molecular analysis were initially ground into powder in liquid nitrogen and were then separately collected for miRNA or protein analyses.

The HEK293T cell line [American Type Culture Collection (ATCC); Manassas, VA, USA] was cultured in Dulbecco's modified Eagle's medium (DMEM) supplemented with $10 \%$ fetal bovine serum (FBS) (both from Gibco, Rockville, MD, USA), $100 \mathrm{U} / \mathrm{ml}$ penicillin and $100 \mathrm{mg} / \mathrm{l}$ streptomycin (Life Technologies, Rockville, MD, USA) in a humidified incubator with $95 \%$ air and $5 \% \mathrm{CO}_{2}$ at $37^{\circ} \mathrm{C}$. The human $\mathrm{CRC}$ cell line HCT-116 (ATCC) and its multidrug-resistant variant HCT-116/L-OHP (established and maintained in our laboratory), were maintained in RPMI-1640 (Gibco) supplemented with $10 \% \mathrm{FBS}, 100 \mathrm{U} / \mathrm{ml}$ penicillin and $100 \mathrm{mg} / 1$ streptomycin in a humidified incubator with $95 \%$ air and $5 \% \mathrm{CO}_{2}$ at $37^{\circ} \mathrm{C}$. To maintain the drug-resistance phenotype, L-OHP (Sigma, St. Louis, MO, USA) was added to the culture medium at a final concentration of $5 \mu \mathrm{g} / \mathrm{ml}$ for HCT-116/L-OHP cells. Chloroquine (CQ; Sigma) was added to the cell culture at a final concentration of $10 \mu \mathrm{M}$.

Quantitative real-time PCR ( $q R T-P C R)$ for miRNA analyses. Total RNA from tissue samples or cells, including miRNAs, was extracted using the miRNA isolation kit (Ambion, Austin, TX, USA) according to the manufacturer's protocol. The purity and concentration of the RNA samples were quantified using a dual-beam ultraviolet spectrophotometer (Eppendorf, Hamburg, Germany). The TaqMan miRNA reverse transcription kit and TaqMan Human miRNA assay kit (both from Applied Biosystems, Foster City, CA, USA) were used to measure miR-218 levels, which were normalized to RNU48 expression. The relative quantification of miR-218 levels was determined using the $2^{-\Delta \Delta \mathrm{Ct}}$ method. The above experiment was performed in triplicate, and each assay included a negative control that lacked cDNA.

Cell transfection. Cultured cells were seeded and transfected with $50 \mathrm{nM}$ corresponding miRNAs, including miR-218, anti-miR-218, control and anti-control mimics (GenePharma, Shanghai, China) using Lipofectamine 2000 reagent (Invitrogen, Carlsbad, CA, USA) in 6- or 96-well plates following the manufacturer's protocol. Transfection efficiency was evaluated by qRT-PCR. After $48 \mathrm{~h}$ of transfection, cells were harvested or processed for further analysis.

To overexpress YEATS4, the YEATS4 coding sequence without the 3'-untranslated region (3'-UTR) was selected and cloned into pcDNA3.1 (Invitrogen), and the empty vector was used as negative control for the detection of transfection efficiency. The plasmids were transfected into cultured cells as indicated using Lipofectamine 2000 according to the manufacturer's instructions.

MTT assay. Cultured cells ( $5 \times 10^{3}$ cells/well) were re-seeded in 96-well plates (Corning Inc., Corning, NY, USA) after transfection with the indicated microRNA mimics or plasmids. After $24 \mathrm{~h}$, the cells were treated with L-OHP for the indicated times, 24,48 or $72 \mathrm{~h}$. Then, $20 \mu \mathrm{l}$ modified 
tetrazolium salt 3-(4,5-dimethyl-2-thiazolyl)-2,5-diphenyl$2 \mathrm{H}$-tetrazolium bromide (MTT; $5 \mathrm{mg} / \mathrm{ml}$; Sigma Aldrich) was added to each well and samples were incubated at $37^{\circ} \mathrm{C}$ for $4 \mathrm{~h}$. The supernatant was then removed and $100 \mu \mathrm{l}$ dimethyl sulfoxide (DMSO; Sigma) was added to each well. After the dark-blue MTT crystals were dissolved, the absorbance was measured at $490 \mathrm{~nm}$ using a Bio-Rad Benchmark Microplate Reader (Bio-Rad, Hercules, CA, USA). The data were defined as: (mean L-OHP-treated $\mathrm{A}_{490}$ - blank)/(mean untreated control $\mathrm{A}_{490}$ - blank) x 100 , and analyzed by weighted non-linear regression to determine the inhibitory concentration $\left(\mathrm{IC}_{50}\right)$. The $\mathrm{IC}_{50}$ value of each cell line to L-OHP was defined as the concentration that inhibited MTT metabolism by $50 \%$ compared with the untreated controls.

Luciferase reporter assay. The potential target of miR-218 was predicted by TargetScan (http://www.targetscan.org) to be YEATS4, which suggests a way to modulate drug resistance through the miRNA level in human CRC. The 3'-UTR of human YEATS4 containing the miR-218 targeting sequence was inserted into the pMIR-REPORT ${ }^{\mathrm{TM}}$ miRNA expression reporter vector system (Ambion). The reporter vector plasmid containing either the YEATS4-wt 3'-UTR or the YEATS4-mut 3'-UTR sequence was subsequently co-transfected with $50 \mathrm{nM}$ of the corresponding miRNAs into HEK293T cells using Lipofectamine 2000. After $48 \mathrm{~h}$ post-transfection, the cells were harvested and lysed for luciferase assays. The luciferase assay kit (Promega, Madison, WI, USA) was used to measure the reporter activity according to the manufacturer's protocol.

Flow cytometric analysis of apoptosis. HCT116/L-OHP or HCT116 cells $\left(4 \times 10^{5}\right.$ cells/well) were plated into 6-well plates and transfected with $50 \mathrm{nM}$ of the corresponding miRNA mimics or pcDNA3.1-YEATS4 plasmids. After $48 \mathrm{~h}$, the cells were treated with 10 or $20 \mu \mathrm{g} / \mathrm{ml} \mathrm{L-OHP.} \mathrm{After} 24 \mathrm{~h}$, the cells were collected, washed twice with ice-cold phosphatebuffered saline (PBS), and then resuspended in $400 \mu \mathrm{l}$ of $1 \mathrm{X}$ binding buffer. After addition of $5 \mu \mathrm{l}$ of Annexin V-FITC, the cells were gently vortexed, and then incubated for $10 \mathrm{~min}$ at $4^{\circ} \mathrm{C}$ in the dark. Propidium iodide (PI; $10 \mu \mathrm{l}$ ) was then added, followed by incubation for another $5 \mathrm{~min}$ at $4^{\circ} \mathrm{C}$ in the dark. Flow cytometry was then conducted using a FACScan flow cytometer (Becton-Dickinson). The fraction of the cell population that was present in different quadrants was analyzed using quadrant statistics. The cells in the lower-right quadrant represented early apoptosis and those in the upper-right quadrant represented late apoptotic cells.

Western blot analysis. Cell lysates were gathered and protein concentrations were determined using the Pierce BCA protein assay kit (Thermo Scientific, Rockford, IL, USA). Equal amounts of protein were processed for western blot analysis following the standard protocols. The primary antibodies used were anti-microtubule-associated protein 1 light chain 3 (LC3) antibody (Novus Biologicals, Littleton, CO, USA); rabbit anti-YEATS4 polyclonal antibody (MyBioSource.com, San Diego, CA, USA); anti- $\beta$-actin antibodies (Santa Cruz Biotechnology, Santa Cruz, CA, USA). After incubation with the appropriate secondary antibody, the resultant protein bands were visualized using enhanced chemiluminescence
ECL (Beyotime, Shanghai, China). The absorbance values of the target proteins were performed through Gel-Pro Analyzer version 4.0 software (Media Cybernetics, Silver Spring, MD, USA), and data were normalized to $\beta$-actin.

Statistical analysis. Data are expressed as mean $\pm \mathrm{SD}$ of the results derived from three independent experiments performed in triplicate. Statistical analysis was performed using the Student's t-test and ANOVA. $\mathrm{P}<0.05$ was considered to indicate statistical significance compared with the respective control.

\section{Results}

miR-218 downregulation and YEATS4 upregulation in CRC tissues and HCT-116/L-OHP cells are associated with L-OHP resistance. Western blot analysis showed that the protein level of YEATS4 was significantly upregulated in both CRC tissues and HCT-116/L-OHP cells compared with this level in the corresponding non-tumor colon tissues and parental HCT-116 cells, respectively (Fig. 1A and B). qRT-PCR showed that the level of miR-218 was lower in both the CRC tissues and HCT-116/L-OHP cells compared with the level in the respective control group (Fig. 1C and D). These data suggest that miR-218 expression was downregulated and YEATS4 expression was upregulated in the CRC tissues and HCT116/L-OHP cells, in association with L-OHP resistance.

miR-218 overexpression sensitizes HCT-116 cells to L-OHP-induced cell apoptosis. To investigate whether miR-218 expression influences L-OHP resistance in HCT-116 and HCT-116/L-OHP cells, we transfected the cells with corresponding mimics followed by L-OHP treatment, and then the MTT assay was performed. The results showed that miR-218 overexpression significantly decreased the $\mathrm{IC}_{50}$ value of L-OHP in the HCT-116/L-OHP cells, and miR-218 suppression significantly enhanced the $\mathrm{IC}_{50}$ value of L-OHP in the HCT-116 cells (Fig. 2A and B). Compared with the control mimic group, miR-218 overexpression alone in the HCT-116/ L-OHP cells elevated the cell apoptosis rate, which was further enhanced in response to L-OHP treatment (Fig. 2C). miR-218 inhibition decreased the HCT-116 cell apoptosis rate following treated with L-OHP (Fig. 2D). These results indicate that miR-218 is capable of sensitizing HCT-116 cells to L-OHP treatment.

miR-218 overexpression increases the sensitivity of HCT-116/ $L-O H P$ cells to $L-O H P$ treatment via inhibition of autophagy. Detection of LC3 is regarded as a common way to monitor autophagy. As LC3 II is rapidly degraded by lysosomal turnover during autophagy, we treated cells with L-OHP in the presence or absence of the lysosome inhibitor CQ. Autophagic flux can be reflected by the fold-change in LC3 II levels with or without CQ $(16,17)$. We found that, compared with the small increase observed in parental L-OHP-sensitive HCT-116 cells, the relative LC3 II levels in L-OHP-resistant HCT-116/L-OHP cells after lysosome inhibition via CQ was markedly upregulated in response to L-OHP treatment, suggesting induction of autophagy (Fig. 3A and B). In addition, exposure of HCT-116/L-OHP cells to L-OHP after control mimic transfection increased autophagic flux, as reflected by 

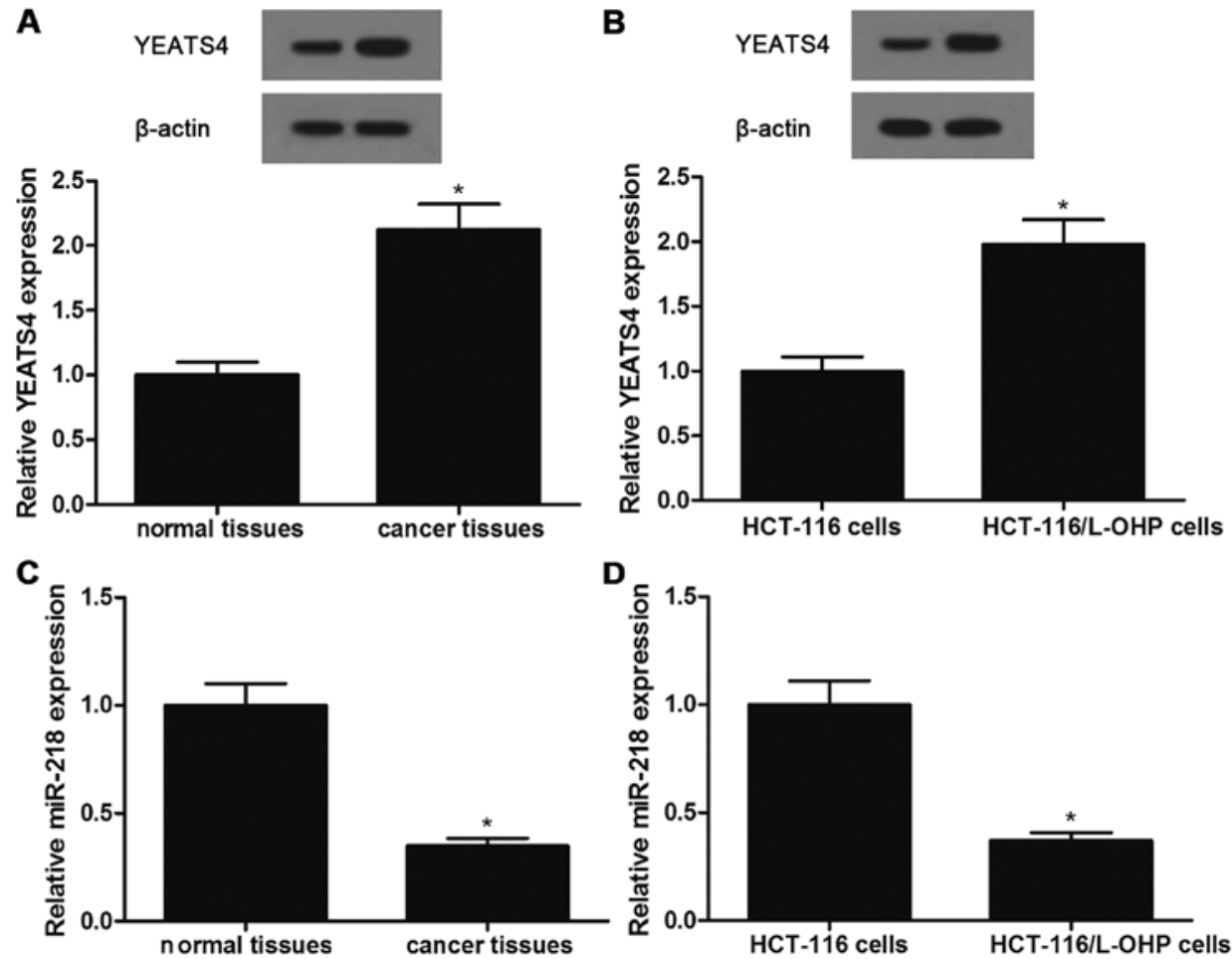

Figure 1. Expression levels of YEATS4 and miR-218 in normal and CRC tissues, as well as HCT-116 and HCT-116/L-OHP cells. (A and B) Relative YEATS4 expression levels were determined by western blotting and representative blots are shown. Quantitative analysis was performed using the Gel-Pro Analyzer version 4.0 software. (C and D) Relative miR-218 expression levels were detected by qRT-PCR; ${ }^{*} \mathrm{P}<0.05$ vs. the normal tissues or the HCT-116 cell group.

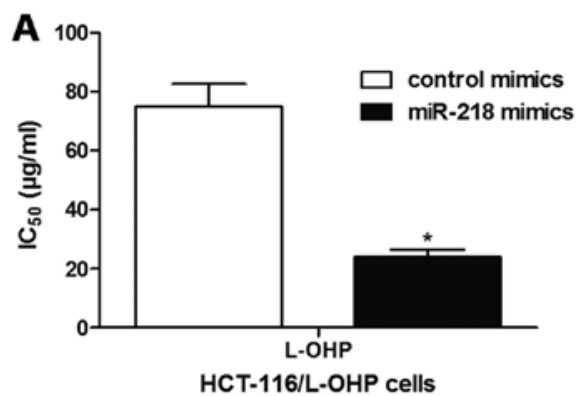

C
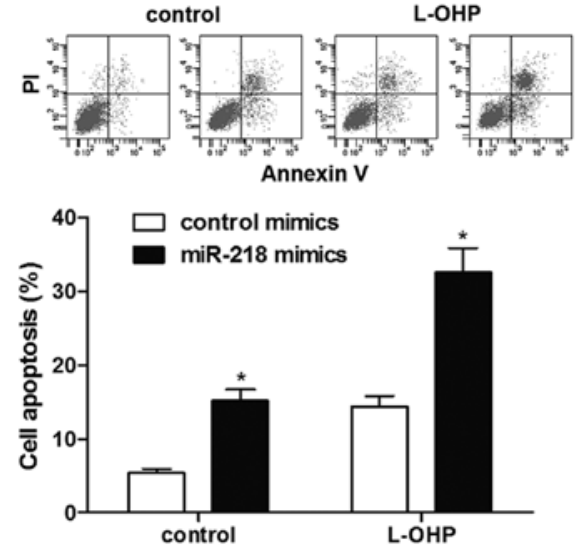

HCT-116/L-OHP cells

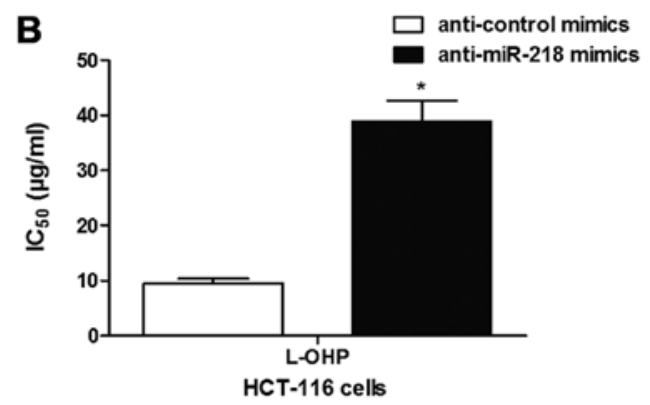

D
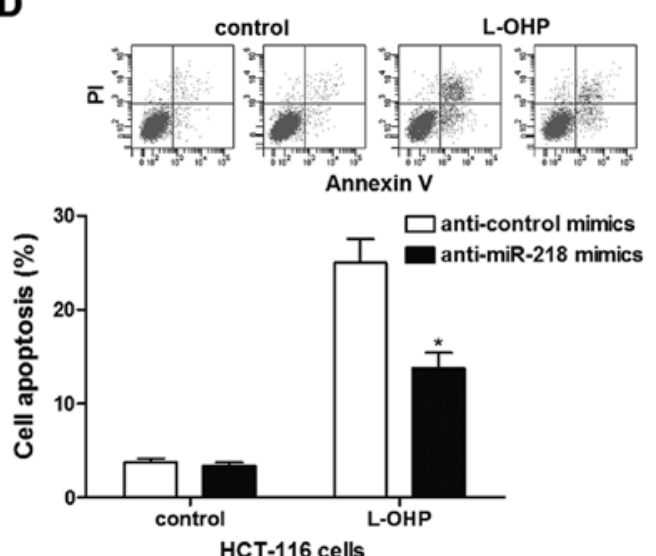

Figure 2. Effects of miR-218 overexpression on $\mathrm{IC}_{50}$ value and cell apoptosis in HCT-116/L-OHP cells in response to L-OHP. (A) HCT-116/L-OHP cells were re-seeded into 96-well plates after transfection with control or miR-218 mimics followed by L-OHP treatment at various concentrations for $72 \mathrm{~h}$. Cell viability was measured by MTT assay. The $\mathrm{IC}_{50}$ value of L-OHP in these cells was presented as mean $\pm \mathrm{SD}$; $\mathrm{P}<0.05$ vs. the control mimic group. (B) HCT-116 cells were re-seeded into 96-well plates after transfection with anti-control or anti-miR-218 mimics followed by L-OHP treatment at various concentrations for $72 \mathrm{~h}$. Cell viability was measured by MTT assay. The $\mathrm{IC}_{50}$ value of L-OHP in these cells was presented as mean \pm SD; "P $<0.05$ vs. the anti-control mimic group. (C) HCT-116/L-OHP cells were treated with $20 \mu \mathrm{g} / \mathrm{ml} \mathrm{L-OHP} \mathrm{for} 24 \mathrm{~h}$ after transfection with control or miR-218 mimics. The cell apoptosis rate was measured by flow cytometric assay; ${ }^{P} \mathrm{P}<0.05 \mathrm{vs}$. the control mimic group. (D) HCT-116 cells were treated with $10 \mu \mathrm{g} / \mathrm{ml} \mathrm{L}-\mathrm{OHP}$ for $24 \mathrm{~h}$ after transfection with anti-control or anti-miR-218 mimics. The cell apoptosis rate was measured by flow cytometric assay; ${ }^{\mathrm{P}}<0.05$ vs. the anti-control mimic group. 
A

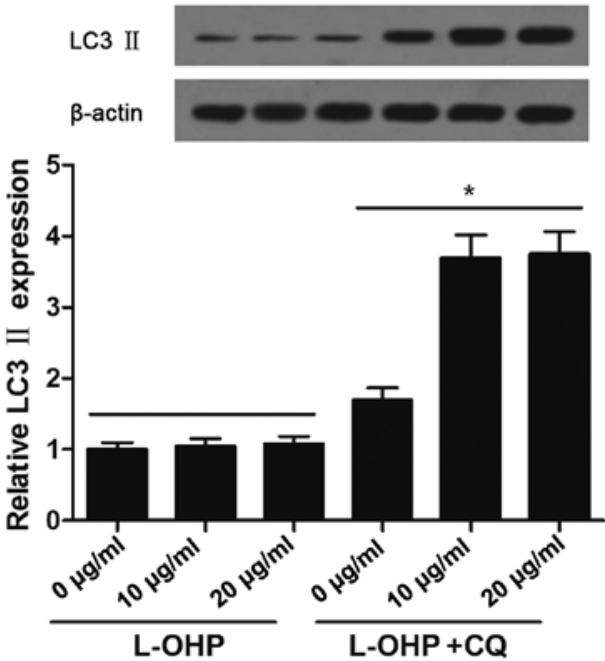

C
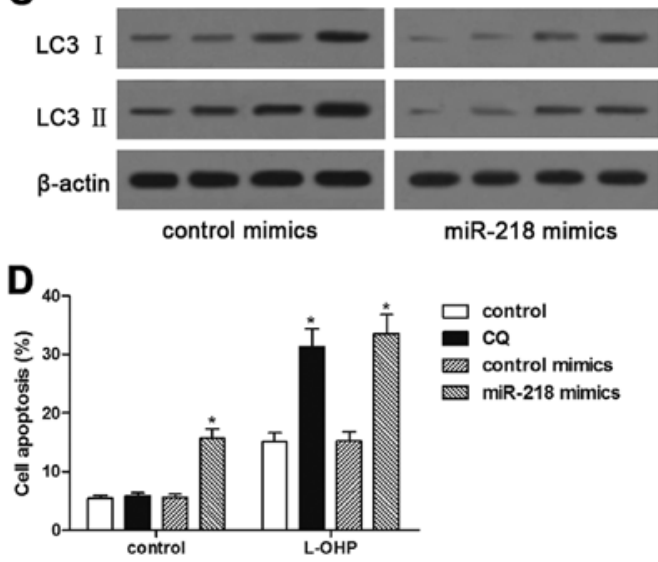

B
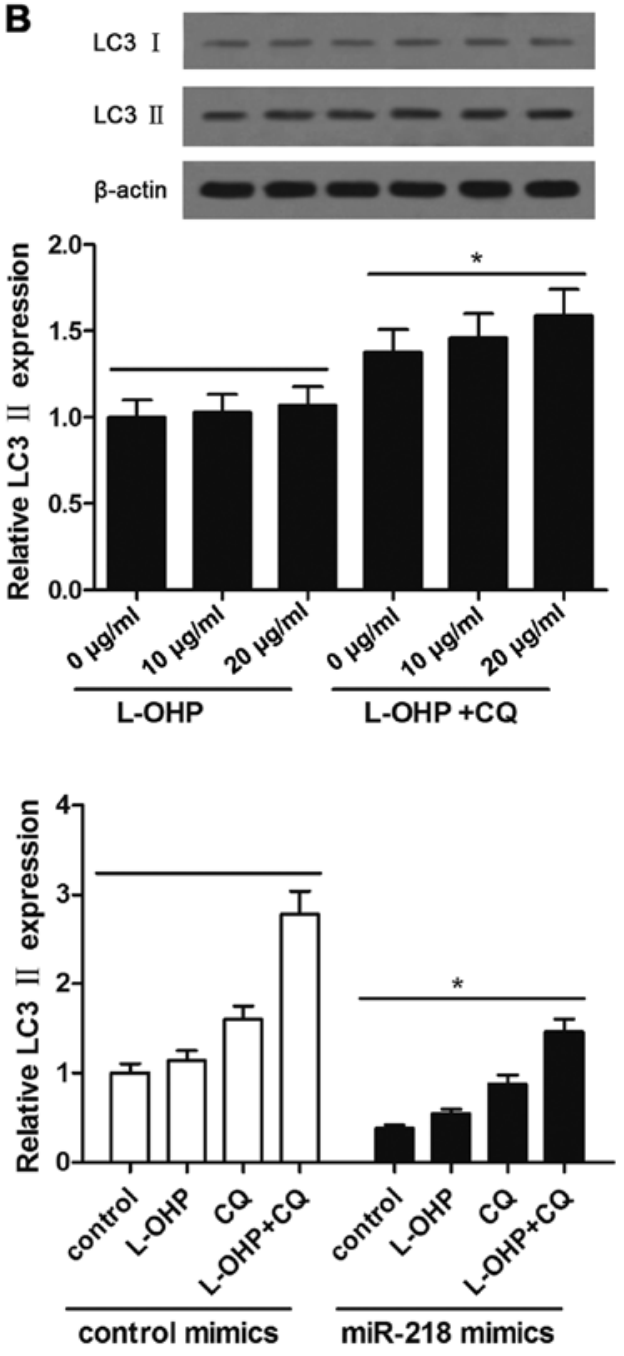

Figure 3. Effects of miR-218 overexpression on cell autophagy and cell apoptosis in HCT-116/L-OHP cells in response to L-OHP. (A) HCT-116/L-OHP or (B) HCT-116 cells were treated with L-OHP at indicated doses $(0,10$ and $20 \mu \mathrm{g} / \mathrm{ml})$ for $24 \mathrm{~h}$ in the presence or absence of $10 \mu \mathrm{M}$ CQ. The expression levels of LC3 II and LC3 I were determined by western blotting and representative blots are shown. The autophagic flux was quantified by the fold-change in LC3 II levels in the presence or absence of CQ; ${ }^{*} \mathrm{P}<0.05$ vs. the L-OHP alone group. (C) HCT-116/L-OHP cells were treated with $20 \mu \mathrm{g} / \mathrm{ml} \mathrm{L}-\mathrm{OHP}$ for $24 \mathrm{~h}$ in the presence or absence of $10 \mu \mathrm{M}$ CQ after transfection with control or miR-218 mimics. The expression levels of LC3 II and LC3 I were determined by western blotting and representative blots are shown; ${ }^{*} \mathrm{P}<0.05 \mathrm{vs}$. the control mimic group. (D) HCT-116/L-OHP cells were treated with $20 \mu \mathrm{g} / \mathrm{ml} \mathrm{L}-\mathrm{OHP}$ for $24 \mathrm{~h}$ in the presence or absence of CQ after transfection with $50 \mathrm{nM}$ control or miR-218 mimics. The cell apoptosis rate was measured by flow cytometric assay; ${ }^{*} \mathrm{P}<0.05$ vs. the control group.

increased LC3 II levels. However, miR-218 overexpression partly reversed L-OHP-mediated LC3 II accumulation in the HCT-116/L-OHP cells (Fig. 3C). To test the hypothesis that $\mathrm{L}-\mathrm{OHP}$ resistance is due to autophagy protection, we treated cells with CQ to determine whether inhibition of autophagy enhances L-OHP-induced apoptosis. Indeed, CQ treatment promoted L-OHP-induced cell apoptosis in the HCT-116/ L-OHP cells, and miR-218 overexpression obtained the same results. These results indicate that $\mathrm{miR}-218$ overexpression increased the sensitivity of HCT-116/L-OHP cells to L-OHP treatment via inhibition of autophagy.

YEATS4 is a direct target of miR-218. To elucidate the underlying mechanisms involving miR-218 and YEATS4, we performed a bioinformatic prediction and the results showed that there was one putative binding site between miR-218 and the 3'-UTR of YEATS4 (Fig. 4A). To confirm the binding, a luciferase reporter assay was performed by evaluating the luciferase activity of HEK293T and HCT-116 cells transfected with pMIR-YEATS4 3'-UTR plasmids and comparing this activity with that of cells transfected with control plasmids. The results showed that miR-218 suppressed the luciferase expression of YEATS4-wt, whereas YEATS4-mut induced no suppressive effect in either HEK293T or HCT-116 cells (Fig. 4B). Western blot analysis indicated that the basal level of YEATS4 in the HCT-116/L-OHP cells was higher than that in the HCT-116 cells, and miR-218 overexpression resulted in a reduction in YEATS4 protein levels in both cells. Furthermore, suppression of miR-218 in HCT-116 cells enhanced the YEATS4 protein level in the HCT-116 cells (Fig. 4C). In addition, L-OHP treatment dose-dependently elevated YEATS4 expression in the HCT-116/L-OHP cells (Fig. 4D). Taken together, these data strongly suggest that miR-218 directly targets YEATS4 expression in both HCT-116 and HCT-116/L-OHP cells. 

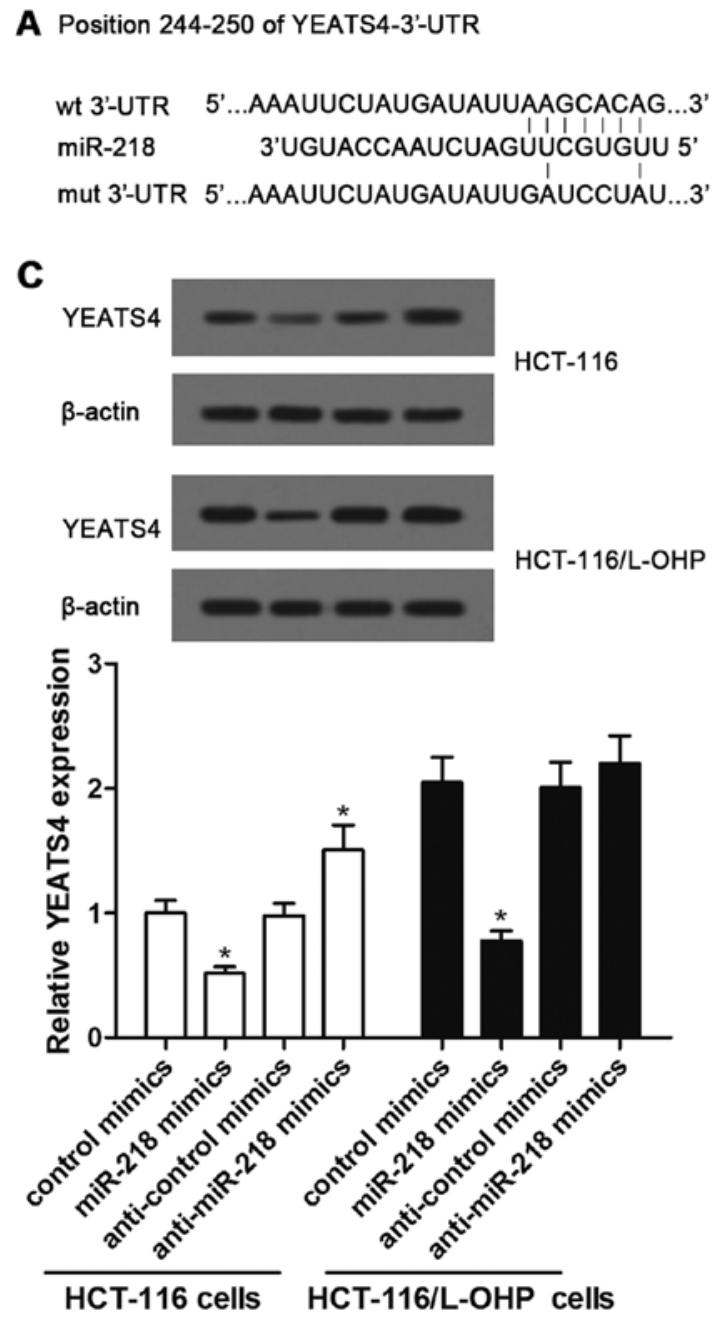

B
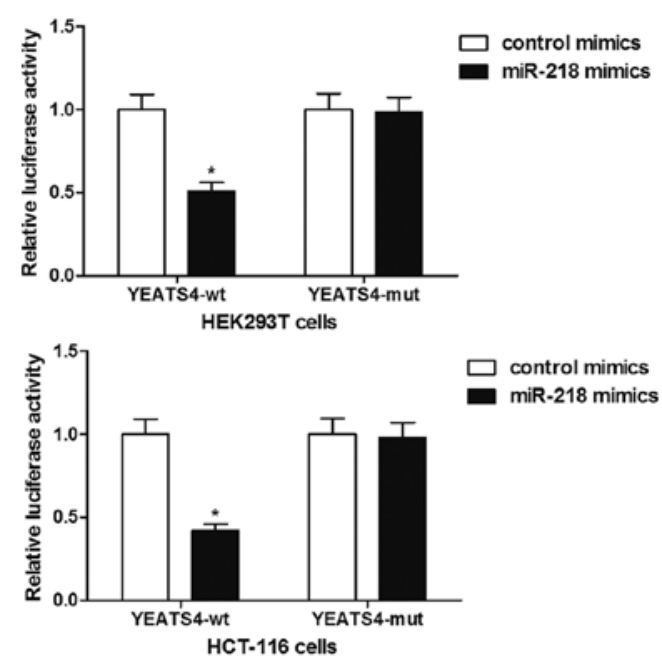

D

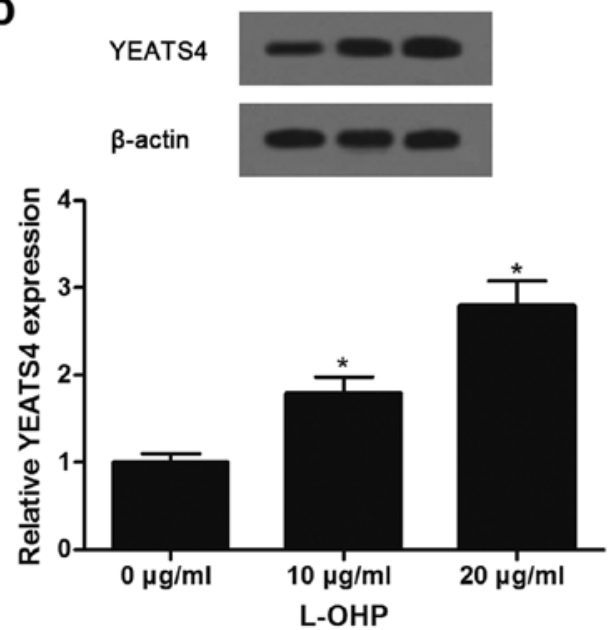

Figure 4. YEATS4 is a direct target gene of miR-218. (A) MicroRNA database search revealed one possible binding site in the YEATS4 3'-UTR for miR-218. (B) Luciferase reporter assay was conducted in HEK293T and HCT-116 cells; " $\mathrm{P}<0.05$ vs. the control mimic group. (C) miR-218 expression levels in HCT-116 and HCT-116/L-OHP cells were determined by qRT-PCR $48 \mathrm{~h}$ after transfection with corresponding miRNAs; ${ }^{*} \mathrm{P}<0.05 \mathrm{vs}$. the control mimic group. (D) YEATS4 protein levels in HCT-116/L-OHP cells were determined by western blotting after treatment with 0,10 or $20 \mu \mathrm{g} / \mathrm{ml} \mathrm{L-OHP} \mathrm{for} 24 \mathrm{~h}$. Quantitative analysis was performed using the Gel-Pro Analyzer version 4.0 software; ${ }^{*} \mathrm{P}<0.05$ vs. the control group.

YEATS4 overexpression reverses the stimulatory effect of miR-218 on cell apoptosis in response to L-OHP via promotion of basal autophagy in HCT-116/L-OHP cells. Western blot analysis indicated that YEATS4 overexpression without the 3'-UTR robustly enhanced the expression of YEATS4, which was significantly reduced by miR-218 overexpression (Fig. 5A). YEATS4 overexpression restored LC3 II expression following reduction by miR-218 in the presence of CQ (Fig. 5B). In addition, YEATS4 overexpression abolished the loss of cell viability and increase in cell apoptosis promoted by miR-218 in response to L-OHP (Fig. 5C and D). Thus, the data confirmed that YEATS4 overexpression reversed the stimulatory effect of miR-218 on cell apoptosis in response to L-OHP by promoting basal autophagy in HCT-116/L-OHP cells.

\section{Discussion}

Colorectal cancer (CRC) is one of the leading causes of cancer-related mortality worldwide. Resistance to chemo- therapy remains the primary reason for treatment failure in advanced CRC patients (3). YEATS4 overexpression has been linked to the aggressive phenotypes of certain types of cancers, including CRC. Several important miRNAs have been identified as regulators of L-OHP treatment in CRC, such as miR-378 (18), miR-21 (19) and miR-222 (20). Therefore, the identification of aberrantly expressed YEATS4 and miRNAs is an important first step towards elucidating YEATS4-mediated or miRNA-mediated oncogenic pathways.

Previous studies have demonstrated that miRNAs are important regulators of carcinogenesis, progression, invasion, angiogenesis and metastases in CRC, which may serve as potential predictive and prognostic factors and even as therapeutic targets themselves. A large body of research has demonstrated that miR-218 is downregulated in multiple malignancies, including glioblastoma (21), nasopharyngeal (22) and lung cancer (23), and CRC (24), and it functions as a tumorsuppressor gene in various cancers targeting many genes to globally regulate biological processes (25-27). Recent progress in cancer biology has also shown that YEATS4 overexpres- 
A
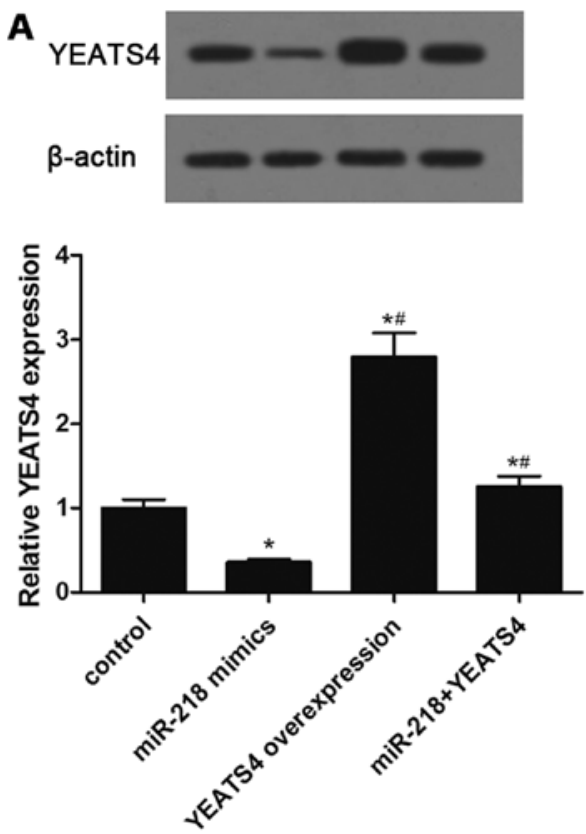

C

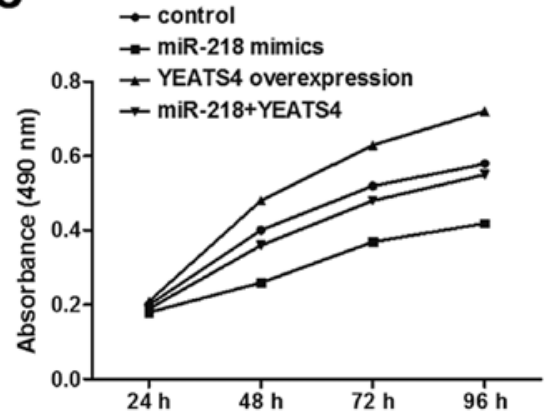

B

LC3 II

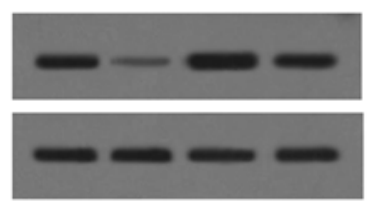

$\beta$-actin
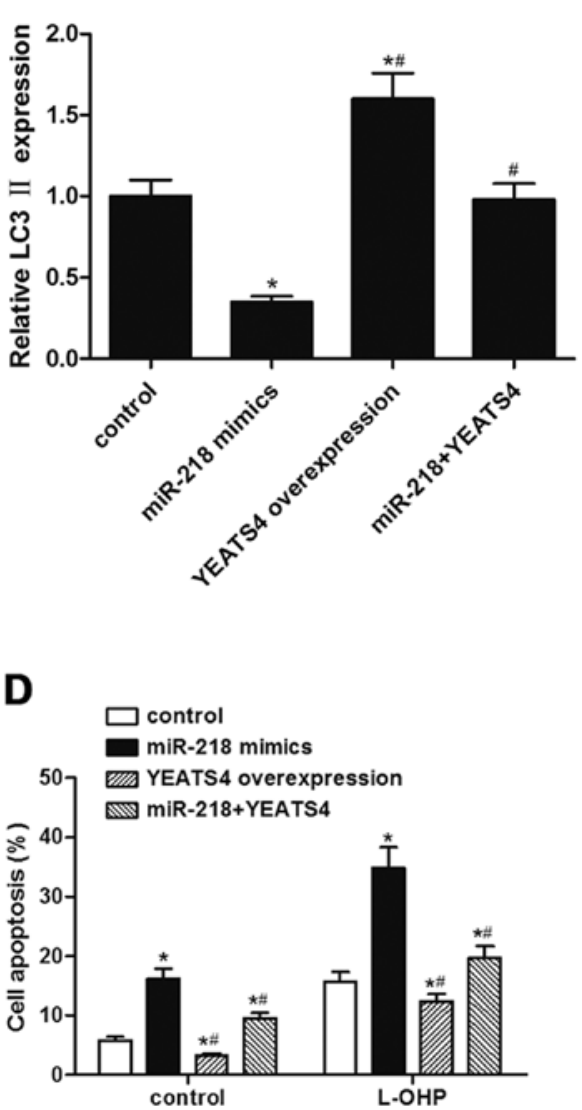

Figure 5. Effects of YEATS4 overexpression on inhibition of basal autophagy and stimulation of apoptosis by miR-218 overexpression in response to L-OHP in HCT-116/L-OHP cells. (A) HCT-116/L-OHP cells were treated with $20 \mu \mathrm{g} / \mathrm{ml} \mathrm{L}$-OHP for $24 \mathrm{~h}$ after transfection with $50 \mathrm{nM}$ miR-218 mimics or pcDNA3.1-YEATS4 plasmids. YEATS4 expression was determined by western blotting and representative blots are shown. (B) HCT-116/L-OHP cells were treated with $20 \mu \mathrm{g} / \mathrm{ml} \mathrm{L-OHP}$ for $24 \mathrm{~h}$ in the presence of $10 \mu \mathrm{M} \mathrm{CQ}$ after transfection with $50 \mathrm{nM}$ miR-218 mimics or pcDNA3.1-YEATS4 plasmids. LC3 II expression was determined by western blotting and representative blots are shown. (C) HCT-116/L-OHP cells were treated with $20 \mu \mathrm{g} / \mathrm{ml} \mathrm{L-OHP} \mathrm{for} 72 \mathrm{~h}$ after transfection with $50 \mathrm{nM}$ miR-218 mimics or pcDNA3.1-YEATS4 plasmids. Cell viability was measured by MTT assay. (D) HCT-116/L-OHP cells were treated with $20 \mu \mathrm{g} / \mathrm{ml} \mathrm{L}-\mathrm{OHP}$ or left untreated for $24 \mathrm{~h}$ after transfection with $50 \mathrm{nM}$ miR-218 mimics or pcDNA3.1-YEATS4 plasmids. The cell apoptosis rate was measured by flow cytometric assay; ${ }^{*} \mathrm{P}<0.05$ vs. the control group; ${ }^{\mathrm{P}} \mathrm{P}<0.05$ vs. the $\mathrm{miR}-218$ mimic group.

sion is observed in some cancers such as non-small cell lung cancer (28), astrocytomas $(11,29)$, glioblastomas (29), liposarcoma (30) and CRC (12). Furthermore, miR-218 enhances 5-fluorouracil-induced apoptosis in CRC (31), and increases the sensitivity of gastric cancer cells to anticancer drugs, including L-OHP (25). Moreover, knockdown of YEATS4 inhibits CRC cell proliferation and induces apoptosis (12). Notably, YEATS4 is implicated as the upstream transcription factor that most often regulates genes implicated in drug resistance $(32,33)$, and $70 \%$ knockdown of YEATS4 siRNA was found to result in $20-30 \%$ recovery of drug sensitivity in ovarian cancer (13). In the present study, we reported that both miR-218 downregulation and YEATS4 upregulation were observed in CRC tissues and HCT-116/L-OHP cells, which were associated with L-OHP resistance. Moreover, miR-218 overexpression sensitized HCT-116 cells to L-OHP-induced cell apoptosis by targeting YEATS4 expression.
Presently, autophagy has been reported to play a complex role at multiple levels of tumor development, but it is generally accepted that autophagy promotes cancer cell survival in the process of tumor development. Autophagy has been considered a mechanism through which cancer cells can survive the stress of damage caused by anticancer therapy and subsequently become resistant to treatment. Our data also showed that L-OHP induced autophagy in HCT-116/L-OHP cells, which was inhibited by miR-218 overexpression. More importantly, autophagy partially results in drug resistance (34), and inhibition of autophagy may enhance the effectiveness of cancer treatment. Furthermore, it is well documented that miRNAs do not only regulate tumor survival and sensitivity to chemotherapy and radiotherapy, but also affect autophagy (35). miR-218 was found to inhibit HMGB1-mediated autophagy in endometrial carcinoma cells during chemotherapy (36). Similarly, the present study showed that overexpression of 
miR-218 increased the sensitivity of HCT-116/L-OHP cells to L-OHP treatment via inhibition of autophagy. Further research revealed that YEATS4 is a novel oncogene amplified in non-small cell lung cancer, which negatively regulates the p53 pathway (28). Inhibition of autophagy exerts anti-CRC effects via apoptosis induced by p53 activation and ER stress (37). We speculated that activation of autophagy was involved in YEATS4 overexpression-mediated cell apoptosis in the HCT-116/L-OHP cells. Indeed, YEATS4 overexpression reversed miR-218-stimulated cell apoptosis in response to L-OHP via promoting basal autophagy in HCT-116/L-OHP cells.

Taken together, the results of the present study highlight the role of miR-218 in the mechanism of chemotherapy sensitivity in CRC, and revealed that miR-218 sensitized HCT-116/L-OHP cells to L-OHP-induced cell apoptosis via inhibition of cytoprotective autophagy by targeting YEATS4 expression. Thus, along with further research into its targets and upstream regulators, miR-218 could be considered as a predictor of L-OHP treatment for personalized treatment, and restoration of miR-218 levels could be a potential novel strategy by which to enhance chemosensitivity to L-OHPbased treatment in the clinic.

\section{References}

1. Stiegelbauer V, Perakis S, Deutsch A, Ling H, Gerger A and Pichler M: MicroRNAs as novel predictive biomarkers and therapeutic targets in colorectal cancer. World J Gastroenterol 20: 11727-11735, 2014.

2. Fang L, Li H, Wang $\mathrm{L}, \mathrm{Hu} \mathrm{J}$, Jin T, Wang $\mathrm{J}$ and Yang BB: MicroRNA-17-5p promotes chemotherapeutic drug resistance and tumour metastasis of colorectal cancer by repressing PTEN expression. Oncotarget 5: 2974-2987, 2014.

3. Longley DB and Johnston PG: Molecular mechanisms of drug resistance. J Pathol 205: 275-292, 2005.

4. Yang HZ, Ma Y, Zhou Y, Xu LM, Chen XJ, Ding WB and Zou HB: Autophagy contributes to the enrichment and survival of colorectal cancer stem cells under oxaliplatin treatment. Cancer Lett 361: 128-136, 2015.

5. Shi Y, Han Y, Xie F, Wang A, Feng X, Li N, Guo H and Chen D: ASPP2 enhances oxaliplatin (L-OHP)-induced colorectal cancer cell apoptosis in a p53-independent manner by inhibiting cell autophagy. J Cell Mol Med 19: 535-543, 2015.

6. Liu W, Zhang Z, Zhang Y, Chen X, Guo S, Lei Y, Xu Y, Ji C, Bi Z and Wang K: HMGB1-mediated autophagy modulates sensitivity of colorectal cancer cells to oxaliplatin via MEK/ERK signaling pathway. Cancer Biol Ther 16: 511-517, 2015.

7. Xie L, Jing R, Qi J, Lin Z and Ju S: Drug resistance-related microRNAs in hematological malignancies: Translating basic evidence into therapeutic strategies. Blood Rev 29: 33-44, 2015.

8. Taniguchi K, Sugito N, Kumazaki M, Shinohara H, Yamada N, Nakagawa Y, Ito Y, Otsuki Y, Uno B, Uchiyama K, et al: MicroRNA-124 inhibits cancer cell growth through PTB1/PKM1/PKM2 feedback cascade in colorectal cancer. Cancer Lett 363: 17-27, 2015.

9. Zhong X, Xiao Y, Chen C, Wei X, Hu C, Ling X and Liu X: MicroRNA-203-mediated posttranscriptional deregulation of CPEB4 contributes to colorectal cancer progression. Biochem Biophys Res Commun 466: 206-213, 2015.

10. Yu H, Gao G, Jiang L, Guo L, Lin M, Jiao X, Jia W and Huang J: Decreased expression of miR-218 is associated with poor prognosis in patients with colorectal cancer. Int J Clin Exp Pathol 6: 2904-2911, 2013.

11. Fischer U, Heckel D, Michel A, Janka M, Hulsebos T and Meese E: Cloning of a novel transcription factor-like gene amplified in human glioma including astrocytoma grade I. Hum Mol Genet 6: 1817-1822, 1997.

12. Tao K, Yang J, Hu Y and Deng A: Knockdown of YEATS4 inhibits colorectal cancer cell proliferation and induces apoptosis. Am J Transl Res 7: 616-623, 2015.
13. Kim YR, Park MS, Eum KH, Kim J, Lee JW, Bae T, Lee DH and Choi JW: Transcriptome analysis indicates TFEB1 and YEATS4 as regulatory transcription factors for drug resistance of ovarian cancer. Oncotarget 6: 31030-31038, 2015.

14. Wei MF, Chen MW, Chen KC, Lou PJ, Lin SY, Hung SC, Hsiao M, Yao CJ and Shieh MJ: Autophagy promotes resistance to photodynamic therapy-induced apoptosis selectively in colorectal cancer stem-like cells. Autophagy 10: 1179-1192, 2014.

15. Yang ZJ, Chee CE, Huang S and Sinicrope FA: The role of autophagy in cancer: Therapeutic implications. Mol Cancer Ther 10: 1533-1541, 2011.

16. Stanton MJ, Dutta S, Zhang H, Polavaram NS, Leontovich AA, Hönscheid P, Sinicrope FA, Tindall DJ, Muders MH and Datta K: Autophagy control by the VEGF-C/NRP-2 axis in cancer and its implication for treatment resistance. Cancer Res 73: 160-171, 2013.

17. Mizushima N, Yoshimori T and Levine B: Methods in mammalian autophagy research. Cell 140: 313-326, 2010.

18. Wang KY, Ma J, Zhang FX, Yu MJ, Xue JS and Zhao JS: MicroRNA-378 inhibits cell growth and enhances L-OHPinduced apoptosis in human colorectal cancer. IUBMB Life 66: 645-654, 2014.

19. Faltejskova P, Besse A, Sevcikova S, Kubiczkova L, Svoboda M, Smarda J, Kiss I, Vyzula R and Slaby O: Clinical correlations of miR-21 expression in colorectal cancer patients and effects of its inhibition on DLD1 colon cancer cells. Int J Colorectal Dis 27: 1401-1408, 2012.

20. Xu K, Liang X, Shen K, Sun L, Cui D, Zhao Y, Tian J, Ni L and Liu J: MiR-222 modulates multidrug resistance in human colorectal carcinoma by down-regulating ADAM-17. Exp Cell Res 318: 2168-2177, 2012.

21. Gao $X$ and Jin W: The emerging role of tumor-suppressive microRNA-218 in targeting glioblastoma stemness. Cancer Lett 353: 25-31, 2014.

22. Alajez NM, Lenarduzzi M, Ito E, Hui AB, Shi W, Bruce J, Yue S, Huang SH, Xu W, Waldron J, et al: MiR-218 suppresses nasopharyngeal cancer progression through downregulation of survivin and the SLIT2-ROBO1 pathway. Cancer Res 71: 2381-2391, 2011.

23. Zarogoulidis P, Petanidis S, Kioseoglou E, Domvri K, Anestakis D and Zarogoulidis K: MiR-205 and miR-218 expression is associated with carboplatin chemoresistance and regulation of apoptosis via Mcl-1 and Survivin in lung cancer cells. Cell Signal 27: 1576-1588, 2015.

24. He X, Dong Y, Wu CW, Zhao Z, Ng SS, Chan FK, Sung JJ and Yu J: MicroRNA-218 inhibits cell cycle progression and promotes apoptosis in colon cancer by downregulating BMI1 polycomb ring finger oncogene. Mol Med 18: 1491-1498, 2013.

25. Zhang XL, Shi HJ, Wang JP, Tang HS and Cui SZ: MiR-218 inhibits multidrug resistance (MDR) of gastric cancer cells by targeting Hedgehog/smoothened. Int J Clin Exp Pathol 8: 6397-6406, 2015.

26. Yamamoto N, Kinoshita T, Nohata N, Itesako T, Yoshino H, Enokida H, Nakagawa M, Shozu M and Seki N: Tumor suppressive microRNA-218 inhibits cancer cell migration and invasion by targeting focal adhesion pathways in cervical squamous cell carcinoma. Int J Oncol 42: 1523-1532, 2013.

27. $\mathrm{Hu} Y, \mathrm{Xu} \mathrm{K}$ and Yagüe E: miR-218 targets survivin and regulates resistance to chemotherapeutics in breast cancer. Breast Cancer Res Treat 151: 269-280, 2015.

28. Pikor LA, Lockwood WW, Thu KL, Vucic EA, Chari R, Gazdar AF, Lam S and Lam WL: YEATS4 is a novel oncogene amplified in non-small cell lung cancer that regulates the p53 pathway. Cancer Res 73: 7301-7312, 2013.

29. Fischer U, Meltzer P and Meese E: Twelve amplified and expressed genes localized in a single domain in glioma. Hum Genet 98: 625-628, 1996.

30. Barretina J, Taylor BS, Banerji S, Ramos AH, Lagos-Quintana M, Decarolis PL, Shah K, Socci ND, Weir BA, Ho A, et al: Subtype-specific genomic alterations define new targets for soft-tissue sarcoma therapy. Nat Genet 42: 715-721, 2010.

31. Li PL, Zhang X, Wang LL, Du LT, Yang YM, Li J and Wang CX: MicroRNA-218 is a prognostic indicator in colorectal cancer and enhances 5-fluorouracil-induced apoptosis by targeting BIRC5. Carcinogenesis 36: 1484-1493, 2015.

32. Park JH and Roeder RG: GAS41 is required for repression of the p53 tumor suppressor pathway during normal cellular proliferation. Mol Cell Biol 26: 4006-4016, 2006. 
33. Carro MS, Lim WK, Alvarez MJ, Bollo RJ, Zhao X, Snyder EY, Sulman EP, Anne SL, Doetsch F, Colman H, et al: The transcriptional network for mesenchymal transformation of brain tumours. Nature 463: 318-325, 2010.

34. Sui X, Chen R, Wang Z, Huang Z, Kong N, Zhang M, Han W, Lou F, Yang J, Zhang Q, et al: Autophagy and chemotherapy resistance: A promising therapeutic target for cancer treatment Cell Death Dis 4: e838, 2013

35. Mikhaylova O, Stratton Y, Hall D, Kellner E, Ehmer B, Drew AF, Gallo CA, Plas DR, Biesiada J, Meller J, et al: VHL-regulated miR-204 suppresses tumor growth through inhibition of LC3Bmediated autophagy in renal clear cell carcinoma. Cancer Cell 21: $532-546,2012$
36. Ran X, Yang J, Liu C, Zhou P, Xiao L and Zhang K: MiR-218 inhibits HMGB1-mediated autophagy in endometrial carcinoma cells during chemotherapy. Int J Clin Exp Pathol 8: 6617-6626, 2015.

37. Sakitani K, Hirata Y, Hikiba Y, Hayakawa Y, Ihara S, Suzuki H, Suzuki N, Serizawa T, Kinoshita H, Sakamoto K, et al: Inhibition of autophagy exerts anti-colon cancer effects via apoptosis induced by p53 activation and ER stress. BMC Cancer 15: 795 , 2015. 